ISSN: 2600-5859

\title{
Análisis estadístico para la elaboración de una bebida natural no convencional a partir de harina de garbanzo (Cicer Arietinum L.) y hojas de muña (Minthostachys Mollys)

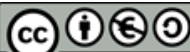

Statistical Analysis for the Preparation of an unconventional natural beverage from chickpea flour (Cicer Arietinum L.) and muña leaves (Minthostachys Mollys)

Jessica Alexandra Marcatoma Tixi. ${ }^{1}$, Diego David Moposita Vásquez. ${ }^{2}$, Paúl Stalin Ricaurte Ortiz. ${ }^{3} \&$ Sonia Lourdes Rodas Espinoza. ${ }^{4}$

Recibido: 05-12-2019 / Revisado: 27-12-2019 / Aceptado: 06-01-2020 / Publicado: 07-02-2020

\begin{abstract}
DOI: https://doi.org/10.33262/concienciadigital.v3i1.1.1158

Non-traditional beverages are products that in recent years tend to raise levels of demand worldwide among consumers, due to the advantages they have compared to common drinks, for this reason the research work was developed with the purpose of create a new consumption option among the range of beverages present in the markets that provide nutrients to the body of those who consume it, as well as avoid diseases, this is how chickpea and muña leaves are among the main raw materials, known for its healing and regenerative properties when combined with the amino acids of the human body. For the preparation of the drink, the characterization of the chickpea flour and muña leaves was carried out through proximal analyzes such as Humidity, Ashes, Fiber, Fat and Protein. Subsequently, the combinations between the concentrations of chickpea flour $(1 \%, 2 \%, 3 \%)$ and muña leaves $(1 \%, 2 \%, 3 \%)$ were obtained, obtaining nine formulations, with three repetitions for each treatment block. After the application of a variance
\end{abstract}

\footnotetext{
${ }^{1}$ Universidad Nacional de Chimborazo, Facultad de Ingeniería, Riobamba, Ecuador, jessica.marcatoma@unach.edu.ec ${ }^{2}$ Universidad Nacional de Chimborazo, Facultad de Ingeniería, Riobamba, Ecuador, dmoposita@unach.edu.ec

${ }^{3}$ Universidad Nacional de Chimborazo, Facultad de Ingeniería, Riobamba, Ecuador, pricaurte@unach.edu.ec

${ }^{4}$ Universidad Nacional de Chimborazo, Facultad de Ingeniería, Riobamba, Ecuador, srodas@unach.edu.ec
} 
analysis with repeated measures, the A6 treatment ( $2 \%$ chickpea flour $+3 \%$ muña leaves) was selected as that combination that maximizes the percentages of Protein and Fiber.

Keywords: Drink, Chickpea, Muña, ANOVA

\section{Resumen}

Las bebidas no tradicionales son productos que durante los últimos años tienden a elevar los niveles de demanda a nivel mundial entre los consumidores, debido a las ventajas que presentan frente a las bebidas comunes, por tal motivo el trabajo de investigación se elaboró con la finalidad de crear una nueva opción de consumo entre la gama de bebidas presentes en los mercados que aporte de nutrientes al organismo de quien lo consume, así como también evitar enfermedades, es así como dentro de las principales materias primas se encuentra el garbanzo y las hojas de muña, conocidas por sus propiedades curativas y regenerativas cuando se combinan con los aminoácidos del cuerpo humano. Para la elaboración de la bebida se realizó la caracterización de la harina de garbanzo y hojas de muña a través de análisis proximales como Humedad, Cenizas, Fibra, Grasa y Proteína. Seguido se postuló a diferentes variantes generadas a partir de la combinación de concentraciones de hojas de muña $(1 \%, 2 \%, 3 \%)$ y harina de garbanzo $(1 \%, 2 \%, 3 \%)$, obteniendo nueve formulaciones, con tres repeticiones por cada bloque de tratamiento. Tras la aplicación de un análisis de varianza con medidas repetidas se seleccionó al tratamiento A6 (2\% de harina de garbanzo $+3 \%$ hojas de muña) como aquella combinación que maximiza los porcentajes de Proteína y Fibra.

Palabras claves: Bebida, Garbanzo, Muña, ANOVA

\section{Introducción}

La ingesta descontrolada de bebidas azucaradas por parte de los habitantes de entre $14 \mathrm{y}$ 64 años de edad, residentes en los diferentes colectivos geográficos ha incrementado la presencia de enfermedades, sobre todo el Diabetes tipo II, Síndrome metabólico y aparición de caries, debido a los elevados niveles de azúcar que la mayoría de refrescos reporta, el antecedente lo ha comprobado un equipo multidisciplinario de investigadores asociados a universidades como Cornell, en Nueva York, a la Emory, en Atlanta donde luego de observar a 13.440 adultos en el estudio REGARDS se detectó que el consumo de bebidas azucaradas y zumos de fruta no contienen porciones significativas de nutrientes para el organismos ya que en ambas presentaciones los elementos que predominan son el agua y el azúcar. Y aunque en los primeros 
el azúcar lo aportan de forma natural las frutas y en los segundos esta se añade en su fabricación, los azúcares específicos que proporcionan al cuerpo son esencialmente los mismos y la respuesta bioquímica cuando se metabolizan es igual. Otro estudio publicado en 'JAMA', concluyen que un elevado consumo de bebidas azucaradas, incluyendo zumos de frutas, se asocian con un aumento de la mortalidad en adultos mayores. Y apoya el hallazgo del equipo del doctor Zhang Yang, que ya demostró en 2014 que aumentar la ingesta de bebidas sin componentes nutricionales eleva el riesgo de mortalidad por problemas cardiovasculares. (Sánchez 2019).

En la actualidad, los estudios de tecnología de alimentos no solo buscan mejorar los productos existentes sino más bien crear nuevos productos basándose en el aporte nutricional para el consumidor, quienes cada vez se encuentran más consciente de la relación que existe entre la dieta y su salud, abriendo un mercado de desarrollo y comercialización de productos con propiedades beneficiosas entre ellas las bebidas elaboradas a partir de materia prima no tradicional, pues estos se encuentran constituidos por elementos biológicamente activos. (Achouri 2007). Es así como la tendencia de la población nacional y mundial hacia el consumo de bebidas naturales no tradicionales ha incrementado los índices de demanda de estos productos al representar uno de los mercados de más rápido crecimiento en el mundo, alcanzando una tasa de incremento anual compuesta del 13.6\% entre 2002 y 2007 (Heckman, MA, 2010). Según la Encuesta Nacional de Salud y Nutrición (2011-2013), el 28 y 35\% de los ecuatorianos consumen bebidas azucaradas y son los hombres quienes consumen con mayor frecuencia en relación a las mujeres. (ENSANUT, 2011), el principal criterio para la aceptación de este tipo de bebidas es el sabor y su composición.

En prevención y corrección a la problemática latente organismos de nutrición promocionan de forma constante el consumo de bebidas naturales no convencionales ya que estas se asocian con la prevención de enfermedades, por lo que las guías alimentarias de los Estados Unidos (USDA), destacan la necesidad del consumo de cereales y leguminosas integrales (USDA, 2010), como parte de los procesos nutricionales diarios, sin embargo, la mayoría de bebidas comerciales son elaboradas a partir de materiales sintéticos que no contribuyen a una alimentación saludable ya que carecen de aporte nutricional significativo.

En tal sentido se pretende utilizar por un lado Cicer arietinum (garbanzo) al considerarlo una leguminosa importante en la nutrición, según (Aguilar \& Vélez, 2013) indicaron que el grano y la harina poseen el 17 y $22 \%$ de proteína respectivamente, (Alfaro, 2016) mencionó la 
existencia de una excelente fuente de fibra, carbohidratos, vitaminas y minerales que ayudan a combatir el colesterol, prevenir el estreñimiento, diabetes además de combatir la celiaquía (Alberca, 2015), por otro lado las hojas de muña (Minthostachys mollys) conocidas medicinalmente por sus propiedades digestivas contra cólicos, vómitos, diarreas y problemas de resfrió; así también es utilizada como analgésico e antiinflamatorio, en tratamientos de tumores y fracturas (Martínez 2019), dentro de las propiedades químicas se puede identificar $\alpha$-pineno, limoneno, carvacrol, timol entre otros. En los países que integran América del sur, es empleada como aromatizante, en la fabricación de licores artesanales y bebidas aromáticas (Huamani, 2015).

La FAO establece que el Cicer arietinum (garbanzo), pertenece a la familia botánica de las leguminosas, muy importantes desde el punto de vista nutricional debido a que son un alimento vegetal ampliamente disponible, que contiene además de carbohidratos una buena cantidad de proteína (Achouri 2007) y vitaminas B1, B2, B9, C, E y K, así como en minerales como calcio, zinc, magnesio, hierro, potasio y fósforo que ayudan a mejorar las defensas del organismo, son una fuente de proteína vegetal. Por cada 100 gramos de alimento aportan unas 350 calorías que se distribuyen en 20 gramos de proteína, 44 de hidratos de carbono, 5 de grasa y 15 de fibra, su aporte de hierro los convierte en un alimento ideal para aquellas personas que padecen de anemia. (Peralta 2014)

Los elementos en estudio son comunes en el estado ecuatoriano gracias a la diversidad de ecosistemas, pisos climáticos y flora muy variada con especies endémicas e insertadas, lo que garantizará la elaboración continua de la bebida de alta calidad, sensorialmente aceptable y con la posibilidad de mejorar la calidad de vida de quienes lo consuman. (Heckman, MA, 2010)

El objetivo de esta investigación fue determinar la mejor combinación entre la harina de garbanzo y hojas de muña entre nueve formulaciones de los compuestos citados donde a través de un estudio exploratorio y con ayuda de la técnica estadística análisis de varianza (ANOVA) se seleccionan las cantidades óptimas para el desarrollo de una bebida natural no convencional.

Mediante la elaboración de la bebida se pretende satisfacer las necesidades y expectativas de los consumidores que se preocupan en el cuidado de su salud. Por otro lado, el sector empresarial tiene la facultad de incorporar en su línea de producción bebidas naturales no convencionales bajo estrictos procesos de calidad y comercializarlos bajo condiciones de un mercado abierto generando considerables beneficios económicos. Además, generar el crecimiento 
ISSN: 2600-5859

WWW.concienciadigital.org

Vol. 3, $\mathrm{N}^{\circ} 1.1$, p. 427-443, febrero, 2020

de emprendimientos ecuatorianos con la creación de microempresas que propongan una nueva línea de producción en torno al consumo de refrescos, los mismos que de ser aceptados tendrían como mercado de consumo potencial a los niños en edades escolares y junto con su demanda nacerá el fortalecimiento de matriz económica y productiva del país.

\section{Metodología}

La presente investigación fue transversal, cuantitativa, experimental, en relación a la línea del tiempo, tipo de investigación y manipulación de variables respectivamente. (Hernández, Fernández y Baptista, 2014) (Patten y Newhart, 2017)

Instrumentos de recolección de datos: La bitácora fue el instrumento que permitió registrar la información de los análisis físico - químicos provenientes de la harina de garbanzo y hojas de muña sin embargo dentro de los materiales utilizados para la elaboración de la bebida se encontraron al pH metro, analizador Kendal VELP.SCIENTHIC para proteína, estufa, balanza analítica y deshidratador DEHYDRATOR, ModelST-02.

Población: El colectivo de estudio estuvo constituido por la producción de harina de garbanzo (Cicer arietinum l.) procesadas en las principales distribuidoras de la cuidad de Riobamba mientras que las hojas de muña (Minthostachys mollys) fueron recolectadas en las zonas altas de la sierra ecuatoriana. (Martínez 2019)

Diseño Muestral: La técnica de selección de datos fue el muestreo aleatorio simple y estuvo representado por la producción de harina de garbanzo procesada por la Asociación SIARIV ubicada en la parroquia de Licán de la ciudad de Riobamba, en cuanto a las hojas de muña se las obtuvo del cantón Chunchi perteneciente a la provincia de Chimborazo. Es importante destacar que el tamaño de la muestra se estableció fijando el error máximo admisible para estimar una proporción y asoció un $95 \%$ de confianza.

Localización del estudio: El estudio se desarrolló en el campus Riobamba de la Universidad de Chimborazo.

Técnicas estadísticas: En cuanto a la necesidad del empleo de técnicas estadísticas se partió por un estudio exploratorio de los resultados provenientes de los análisis físico químicos de la harina de Garbanzo (Cicer Arietinum L.) y hojas de muña (Minthostachys mollys) (Martínez 2019), para la elaboración de la bebida con las mejores características encontradas en función de los parámetros de Humedad, Cenizas, Grasas, Fibra y Proteína, se evaluaron 9 tratamientos (A1: 
ISSN: 2600-5859

$1 \% \mathrm{HG}-1 \% \mathrm{HM}, \mathrm{A} 2: 1 \% \mathrm{HG}-2 \% \mathrm{HM}, \mathrm{A} 3: 1 \% \mathrm{HG}-3 \% \mathrm{HM}, \mathrm{A} 4: 2 \% \mathrm{HG}-1 \% \mathrm{HM}, \mathrm{A} 5: 2 \%$ HG - 2\% HM, A6: 2\% HG - 3\% HM, A7: 3\% HG - 1\% HM, A8: 3\% HG - 2\% HM, A9: 3\% HG - 3\% HM) cada uno de ellos con tres réplicas por lo que se utilizó un ANOVA con medidas repetidas, el uso de la técnica se justifica debido a la naturaleza de la información.

El procedimiento experimental controló los niveles de calidad de la materia prima y de las bebidas según las normativas INEN

\section{Tabla 1}

Normativa de Control INEN

\begin{tabular}{lc}
\hline & Normativa de Control INEN \\
\hline PH & Técnica - NTE INEN 389: 1980. \\
Acidez & Titulación acido-base NTE INEN -0521 \\
Humedad & Estufa - NTE INEN -0518 \\
Cenizas & Mufla- NTE INEN -0520 \\
Grasa & Método Soxhlet -NTE INEN - 0523 \\
Proteína & Método Kjeldhal - NTE INEN - 0519 \\
Fibra & Método gravimétrico- NTE INEN-0522 \\
Aerobios Mesofilos & Técnica- NTE INEN 1529-5:2006. \\
Mohos y Levaduras & Técnica - NTE INEN 1529-10:2013 \\
Escherichia Coli & Técnica - 1529-8 1990. \\
\hline
\end{tabular}

Elaborado por: Grupo de Investigación INVAGRO.

\section{Resultados}

En torno a la clara preocupación de la sociedad por la posible relación entre el estado de salud personal y la alimentación que se recibe, el mercado de alimentos ha incluido en los últimos años productos que anuncian beneficios para la salud, (USDA, 2010) junto con esta visión se procedió a la elaboración de una bebida natural no tradicional cuyo procedimiento inició tras el análisis físico químico de la materia prima: 
ISSN: 2600-5859

\section{Tabla 2}

Análisis físico químico de la harina de Garbanzo

\begin{tabular}{cccccc}
\hline Estadísticos & Humedad (\%) & $\begin{array}{c}\text { Cenizas } \\
(\%)\end{array}$ & Grasa (\%) & Fibra (\%) & $\begin{array}{c}\text { Proteína } \\
(\%)\end{array}$ \\
\hline Media & 7,73 & 0,06 & 14,31 & 5,83 & 15,31 \\
Desviación estándar & 0,026 & 0,001 & 0,03 & 0,03 & 0,045 \\
Coef. Variación & 0,003 & 0,017 & 0,002 & 0,005 & 0,003
\end{tabular}

Elaborado por: Grupo de Investigación INVAGRO. (Martínez 2019)

Para la harina de garbanzo el porcentaje promedio de humedad fue de 7.73 , en cuanto a cenizas fue de 0,06 , las grasas por su parte representaron un porcentaje promedio de 14,31, según la fibra su media fue de 5,83\%, finalmente el porcentaje promedio de proteína fue de 15,31. Los valores referidos de desviación indican la presencia de mediciones homogéneas ya que no existe un grado de dispersión significativa con respecto a la media de las mediciones, esto lo confirmó el coeficiente de variación al indicar que las variables analizadas albergan mediciones semejantes ya que todos los valores son inferiores al 5\%. Mencionados resultados fueron comparados con la normativa INEN para harinas de trigo y se evidenció que los valores hallados fueron inferiores al $15 \%, 3,06 \%$ y $51 \%$ que corresponden a los límites de calidad máximo de Humedad, Cenizas y Fibra, en cuánto a la proteína su valor máximo es de 57\% según (Herrera 2014), sin embargo, se observó un porcentaje elevado de grasa

Tabla 3

Análisis físico químico de las hojas de Muña

\begin{tabular}{cccccc}
\hline Estadísticos & Humedad (\%) & $\begin{array}{c}\text { Cenizas } \\
(\%)\end{array}$ & Grasa (\%) & Fibra (\%) & $\begin{array}{c}\text { Proteína } \\
(\%)\end{array}$ \\
\hline Media & 10,56 & 0,08 & 1,35 & 54,31 & 10,27 \\
Desviación estándar & 0,04 & 0,01 & 0,04 & 0,04 & 0,05 \\
Coef. Variación & 0 & 0,08 & 0,03 & 0 & 0 \\
\hline
\end{tabular}

Elaborado por: Grupo de Investigación INVAGRO.

El porcentaje promedio de humedad hallado en las hojas de muña fue de 10.56 , en cuanto a cenizas fue de 0,08 las grasas por su parte representaron un porcentaje promedio de 1,35, según la fibra su media fue de 54,31\%, finalmente el porcentaje promedio de proteína fue de 10,27. Los 
ISSN: 2600-5859

valores referidos de desviación indican la presencia de mediciones homogéneas ya que no existe un grado de dispersión significativa con respecto a la media de las mediciones, esto lo confirmó el coeficiente de variación al indicar que las variables analizadas albergan mediciones inferiores al $10 \%$.

Al comparar las propiedades de las materias primas se pudo apreciar que en términos de porcentajes de humedad y fibra son las hojas de muña las que mayor valor aportan por otro lado la harina de garbanzo muestra porcentajes elevados entre cenizas, grasas y proteínas lo que evidencia el aporte nutricional de los componentes en el producto.

La materia prima fue mezclada en nueve tratamientos derivantes de la combinación de 1, 2 y $3 \%$ de harina de garbanzo y hojas de muna, cada una de las combinaciones contó con tres réplicas lo que a término del procedimiento permitió obtener 27 bebidas naturales.

\section{Elaboración de la bebida}

En $300 \mathrm{ml}$ de agua purificada sin gas a temperatura de $80{ }^{\circ} \mathrm{C}$, se adicionó harina de garbanzo hasta conseguir una mezcla homogénea en sus diferentes concentraciones, seguido se añadió hojas de muña, las soluciones formuladas reposaron durante cinco minutos en recipientes herméticos, a término del proceso se envasaron en recipientes de cristal los cuales fueron sometidos a pasteurización $\left(70{ }^{\circ} \mathrm{C}\right.$ durante 20 minutos) y posterior choque térmico, con el fin de eliminar microorganismos patógenos presentes en el producto, seguido se procedió a la comparación de variables físico químicas del proceso como: Humedad, Cenizas, Proteínas, Grasas y Fibra para seleccionar la mejor formulación de aporte nutricional de la bebida.

\section{Diseño Experimental}

\section{Tabla 4}

ANOVA para Humedad

\begin{tabular}{lccccc}
\hline Fuente de Variación & SC & Gl & CM & F & p-valor \\
\hline Modelo & 19,32 & 8 & 2,41 & 15,14 & $1 \mathrm{E}-04$ \\
Formulaciones & 19,32 & 8 & 2,41 & 15,14 & $1 \mathrm{E}-04$ \\
Error & 2,87 & 18 & 0,16 & & \\
Total & 22,19 & 26 & & &
\end{tabular}

Elaborado por: Grupo de Investigación INVAGRO. (Martínez 2019)

SC: Suma de cuadrados; gl: Grados de libertad; CM: Cuadrados medios 
ISSN: 2600-5859

Se observó diferencia estadística altamente significativa en el modelo y en las formulaciones ya que el valor de probabilidad asociado es inferior al nivel de significancia del $1 \%$ lo que indica que el porcentaje promedio de humedad es diferente en uno de las nueve formulaciones de harina de garbanzo y hojas de muña, según las comparaciones de Tukey es la formulación (A7: 3\% de garbanzo + 1\% de muña) la que mayor porcentaje de humedad presentó.

\section{Tabla 5}

ANOVA para Cenizas

\begin{tabular}{cccccc}
\hline $\begin{array}{c}\text { Fuente de } \\
\text { Variación }\end{array}$ & SC & Gl & CM & F & p-valor \\
\hline Modelo & 59,69 & 8 & 7,46 & 1676,06 & 0,0001 \\
Formulaciones & 59,69 & 8 & 7,46 & 1676,06 & 0,0001 \\
Error & 0,080 & 18 & 0,004 & & \\
Total & 59,77 & 26 & & & \\
\hline
\end{tabular}

SC: Suma de cuadrados; gl: Grados de libertad; CM: Cuadrados medios

Elaborado por: Grupo de Investigación INVAGRO. (Martínez 2019)

La diferencia estadística se mantiene en el modelo y en las formulaciones al $1 \%$ de significancia para la presencia del porcentaje promedio de cenizas en las tres formulaciones de harina de garbanzo y hojas de muña pues según las comparaciones de Tukey es la formulación (A8: 3\% de garbanzo + 2\% de muña) la que menor porcentaje de cenizas almacenó.

\section{Tabla 6}

ANOVA para Proteínas

\begin{tabular}{cccccc}
\hline $\begin{array}{c}\text { Fuente de } \\
\text { Variación }\end{array}$ & SC & Gl & CM & F & p-valor \\
\hline Modelo & 0,67 & 8 & 0,083 & 319,09 & 0,0001 \\
Formulaciones & 0,67 & 8 & 0,083 & 319,09 & 0,0001 \\
Error & 0,004 & 18 & 0,0003 & & \\
Total & 0,68 & 26 & & & \\
\hline
\end{tabular}

SC: Suma de cuadrados; gl: Grados de libertad; CM: Cuadrados medios

Elaborado por: Grupo de Investigación INVAGRO. (Martínez 2019) 
ISSN: 2600-5859

En el porcentaje promedio de proteínas se evidenció diferencias estadísticas similares a los casos anteriores y se observó según las comparaciones de Tukey que la formulación (A6: 2\% de garbanzo $+3 \%$ de muña) elevó la presencia de proteína en la bebida funcional.

\section{Tabla 7}

ANOVA para Grasas

\begin{tabular}{cccccc}
\hline $\begin{array}{c}\text { Fuente de } \\
\text { Variación }\end{array}$ & SC & Gl & CM & F & p-valor \\
\hline Modelo & 0,60 & 8 & 0,08 & 16,88 & 0,0001 \\
Formulaciones & 0,60 & 8 & 0,08 & 16,88 & 0,0001 \\
Error & 0,08 & 18 & 0,004 & & \\
Total & 0,68 & 26 & & & \\
\hline
\end{tabular}

SC: Suma de cuadrados; gl: Grados de libertad; CM: Cuadrados medios

Elaborado por: Grupo de Investigación INVAGRO. (Martínez 2019)

El porcentaje promedio de grasas también evidenció diferencias estadísticas tanto en el modelo como en las formulaciones y según las comparaciones de Tukey que la formulación A8 ( $3 \%$ de garbanzo $+2 \%$ de muña) contuvo menor contenido de grasa en la bebida funcional.

\section{Tabla 8}

ANOVA para Fibras

\begin{tabular}{cccccc}
\hline $\begin{array}{c}\text { Fuente de } \\
\text { Variación }\end{array}$ & SC & Gl & CM & F & p-valor \\
\hline Modelo & 82,29 & 8 & 10,29 & 329,30 & 0,0001 \\
Formulaciones & 82,29 & 8 & 10,29 & 329,30 & 0,0001 \\
Error & 0,56 & 18 & 0,03 & & \\
Total & 82,85 & 26 & & & \\
\hline
\end{tabular}

SC: Suma de cuadrados; gl: Grados de libertad; CM: Cuadrados medios

Elaborado por: Grupo de Investigación. (Martínez 2019) 
ISSN: 2600-5859

El porcentaje promedio de fibra también presentó diferencias significativas entre los tratamientos y a partir de las comparaciones de Tukey se apreció que la formulación (A6: 2\% de garbanzo y 3\% de muña) almacenó mayor cantidad de fibra. Seguido se procedió al análisis microbiológico de las diferentes combinaciones

Tabla 9

Análisis Microbiológico

\begin{tabular}{ccccccc}
\hline Formulaciones & $\begin{array}{c}\text { Escherichia } \\
\text { Coli } \\
\text { UFC/ml }\end{array}$ & $\begin{array}{c}\text { Escherichia } \\
\text { Coli } \\
\text { UFC/ml }\end{array}$ & $\begin{array}{c}\text { Mohos y } \\
\text { levaduras } \\
\text { UFC/ml }\end{array}$ & $\begin{array}{c}\text { Mohos y } \\
\text { levaduras } \\
\text { UFC/ml }\end{array}$ & $\begin{array}{c}\text { Aerobios } \\
\text { Mesofilos } \\
\text { UFC/ml }\end{array}$ & $\begin{array}{c}\text { Aerobios } \\
\text { Mesofilos } \\
\text { UFC/ml }\end{array}$ \\
\hline A1 & 10 & 8 & 7 & 5 & 15 & 12 \\
A2 & 12 & 5 & 8 & 4 & 18 & 10 \\
A3 & 6 & 2 & 5 & 3 & 20 & 11 \\
A4 & 7 & 3 & 4 & 3 & 25 & 9 \\
A5 & 9 & 6 & 6 & 2 & 23 & 8 \\
A6 & 10 & 9 & 8 & 6 & 22 & 7 \\
A7 & 8 & 5 & 7 & 5 & 31 & 15 \\
A8 & 4 & 2 & 5 & 3 & 23 & 17 \\
A9 & 2 & 1 & 3 & 2 & 22 & 7
\end{tabular}

Elaborado por: Grupo de Investigación. (Martínez 2019)

La presencia de UFC de Escherichia Coli para la dilución se presentó en igual magnitud en las formulaciones A1B1 y A2B3 (10 UFC) sin embargo en la formulación (A2: \% de garbanzo y $1 \%$ de muña) existió mayor presencia de UFC (12 UFC) por otra parte la formulación con menor presencia de UFC fue la combinación A3, en relación a la dilución las formulaciones con mayor y menor presencia de UFC fueron A2 y A3 respectivamente.

En cuanto a los Mohos y Levaduras provenientes de la dilución se evidenció presencia mayoritaria en las formulaciones A1 Y A2 con la misma cantidad de UFC (8) y tan solo 3 UFC en la formulación A3, con respecto a la dilución 6 UFC se encontraron en la formulación A2 y apenas 2 UFC en la formulación A3. 
ISSN: 2600-5859

Las UFC de Aerobios Mesófilos resultantes de la dilución en mayor cantidad fueron hallados en la formulación A3 y su presencia mínima en la combinación A1, lo opuesto ocurre cuando se cambia de dilución, 17 UFC aparecieron en la combinación A3 y 7 UFC en las formulaciones A2 y A3.

Hay que destacar que los análisis microbiológicos fueron realizados también para un nivel de dilución con la normativa INEN y no se encontraron Unidades Formadoras de Colonias de Aerobios Mesófilos, Mohos y Levaduras y Escherichia Coli

Tabla 10

Colonias de Microorganismos

\begin{tabular}{cccc}
\hline Microorganismos & UFC & Método/ Norma & NTE INEN 2337: 2008 \\
\hline Aerobios mesófilos UFC/ml & N/D & INEN 1529-5:2006 & ------ \\
Mohos y levaduras UFC/ml & N/D & INEN 1529-10:2013 & \\
Escherichia Coli & N/D & INEN 1529-8:1990 & \\
UFC/ml & & & \\
\hline
\end{tabular}

Elaborado por: Grupo de Investigación. (Martínez 2019)

Los resultados microbiológicos de la bebida fueron comparados con la NORMA TÉCNICA ECUATORIANA NTE INEN 2337:2008 para jugos, pulpas, concentrados, néctares, bebidas de frutas y vegetales.

Según los resultados obtenidos, en la caracterización de harina de garbanzo se determinó que el $7.73,0.06,14.31,5.83$ y $15.31 \%$ corresponden a los porcentajes de humedad, cenizas, grasa, fibra y proteína respectivamente, citados valores muestran la misma tendencia que los hallados por (Lara, 2016) mientras caracterizaba la harina de garbanzo para la elaboración de pan de molde mostrando el 10.58, 3.61, 6.1, 1.50 y $19.99 \%$ de humedad, cenizas, grasas, fibra y proteína respectivamente.

Por otro lado, en la valoración de las hojas de muña los datos reportados coinciden con la información hallada por (Copacondori \& Nuñez, 2017) quienes realizaron la caracterización de la hoja para elaborar una bebida funcional, dentro de sus análisis encontramos los siguientes resultados $10,66 \%$ en humedad, $5,76 \%$ cenizas, $8,66 \%$ grasa, $17,26 \%$ fibra, $8,24 \%$ proteína. 
Además, al no existir una norma específica para esta materia prima se comparó los datos con la NORMA TÉCNICA ECUATORIANA 2392:2016 para hiervas aromáticas, en la norma encontramos el límite máximo solo de humedad de un $12 \%$ y cenizas de un $2.5 \%$, esto determina a que las mediciones halladas están dentro de los parámetros establecidos tanto de los autores y las normativas.

\section{Conclusiones}

- Al comparar las características fisicoquímicas y microbiológicas de la bebida entre los nueve tratamientos se determinó que las combinaciones con menor y mayor contenido de humedad, fueron A7 (3\% de garbanzo y 1\% de muña) con 94,01\% y A1 (1\% garbanzo y $1 \%$ de muña) con 96,69\%. Según Quezada, 2014, manifiesta que al desarrollar una bebida funcional con garbanzo dentro de los ingredientes se presenta un $81 \%$ de humedad, y según la NORMA TÉCNICA ECUATORIANA 2304:2017 para bebidas no carbonatadas, cualquier valor superior a la cifra referida se considera como presencia de control y calidad.

- En el contenido de cenizas se determinó el tratamiento A8(3\% de garbanzo y 2\% de muña) mostró la presencia del elemento en un 2,62\%. Este resultado se comparó con el estudio realizado por (Copacondori \& Núñez, 2017) quienes elaboraron una bebida funcional a base de malta y hojas de muña, donde se identificó $0,08 \%$ de cenizas, esto determina que la bebida en estudio tiene mayor cantidad de sólidos disueltos o mayor cantidad de minerales. En cuanto a la proteína el tratamiento A6 (2\% de garbanzo y 3\% de muña) maximizó su concentración en un 1,03\%, valor comparado con el estudio de (Copacondori \& Núñez, 2017) donde se desarrolló una bebida funcional a base de malta y hojas de muña el porcentaje de proteína fue de $0,0 \mathrm{~g} / 100 \mathrm{~g}$, aseverando que la bebida desarrollada en la presente investigación tiene mayor cantidad de proteína.

- En la determinación de grasa, el tratamiento A8 (3\% de garbanzo y 2\% de muña) presentó el valor más alto con 1,40\%. Los datos se compararon con (Copacondori \& Núñez, 2017) quienes determinan 0,00\% de grasa en su bebida, esto quiere decir que la bebida en estudio tiene mayor contenido de grasa, debido a la materia prima utilizada como la harina de garbanzo. En el contenido de fibra se determinó que el tratamiento A6 (2\% de garbanzo y $3 \%$ de muña) maximizó su valor con un 3,35\%. Los datos obtenidos se compararon con 
ISSN: 2600-5859

investigaciones relevantes quienes reportan valores de $0,0 \%$ en fibra, demostrando que la bebida desarrollada tiene un contenido mayor en este componente.

- Para el análisis de pH y acidez se utilizó la NORMA TÉCNICA ECUATORIANA 2304:2017 como referencia "Bebidas que contengan aditivos alimentarios como conservantes, colorantes, potenciadores de sabor, acidificantes etc.", misma que especifica un límite de $\mathrm{pH}$ de 4,5\%. El pH promedio de las bebidas en estudio fue de 6,70\% explicando que no es una bebida ácida, no contienen potenciadores de sabor ni acidificantes, pero conservante como el sorbato de potasio. La norma técnica INEN no específica un límite máximo para la acidez de las bebidas, sin embargo, en el estudio se encontró una acidez $1,22 \%$.

- Por tanto, se elige como combinación ideal para la elaboración de la bebida el tratamiento A6: $2 \%$ de garbanzo + 3\% de muña si se busaca elevar el porcentaje de proteínas y fibras dentro del valor nutricional del refresco.

- Finalmente, en los análisis microbiológicos para Escherichia Coli, mohos y levaduras, y aerobios mesófilos comparadas con NORMA TÉCNICA ECUATORIANA NTE INEN 2337:2008. Para "JUGOS, PULPAS, CONCENTRADOS, NECTARES, BEBIDAS DE FRUTAS Y VEGETALES”, se determinó que todos los tratamientos se encuentran dentro del rango establecido.

\section{Referencias bibliográficas}

Aguilar, R., \& Vélez, R. (2013). Propiedades nutricionales y funcionales del garbanzo. Departamento de Ingeniería Química, Alimentos y Ambiental, Universidad de las Américas Puebla, 26-30. Recuperado el 25 de Julio de 2019, de file:///E:/ /anteproyecto\%20tesis/garbanzo.pdf

Alberca, M. R. (2015). "Elaboración y evaluación nutricional de un cupcake a base de harina de achira (Canna_edulis) Fortificado con Harina de Garbanzo (Cicer arietinum 1) y papaya (Carica papaya)". Riobamba - Ecuador: Escuela Superior Politécnica de Chimborazo Facultad de Ciencias.

Alfaro, M. D. (2016). Elaboración de tres embutidos a base de Soja texturizada y Garbanzo. Tuxtla Gutiérrez, Chiapas: Universidad de Artes y Ciencias de Chiapas.

Alkire, B. H., Tucker, A. O., \& Maciarello, M. J. (1994). Tipo Minthostachys Mollis (LAMIACEAE): An Ecuadorian Mint. The New York Botanical Garden, Bronx, 60-64. 
ISSN: 2600-5859

Bruzos, S. C., Candela, C. G., Nomdedeu, C. L., \& Bardonada, M. Á. (2012). Nutrición, salud y alimentos funcionales (Vol. III). (l. o. agueda, Ed.) Madrid, Madrid, España: UNED. Recuperado el 23 de Julio de 2019, de https://books.google.com.ec/books?hl=es\&lr=\&id=hfQMXBIiydgC\&oi=fnd\&pg=PA11 $\& \mathrm{dq}=$ bebidas+funcionales\&ots $=\_X 59 \mathrm{CxDg} Y E \&$ sig $=\mathrm{oKxhk} 2 \mathrm{wZwgETuLfYvFIXf1ktXK}$ $0 \# \mathrm{v}=$ onepage $\& \mathrm{q}=$ bebidas $\% 20$ funcionales $\& \mathrm{f}=$ false

Constitución, d. 1. (2008). Lexisfinder. Obtenido de Lexisfinder: https://www.comunicacion.gob.ec/wp-content/uploads/downloads/2017/03/ConstitucionPolitica-del-Ecuador.pdf

Copacondori, C. E., \& Núñez, D. P. (2017). Evaluación de los parámetros para la obtención de una bebida funcional, A partir de Malta (hordeum vulgare) y Muña (Minthostachys mollys). Arequipa: Universidad Nacional de San Agustín Facultad de Ingeniería de Procesos.

Espeche, C. M., Vizgarra, O. N., Mamani, S. Y., Méndez, D., \& Ploper, L. D. (2000). El Garbanzo una buena Opción para el invierno. DISSIER Avance Agroindustrial, 26. Recuperado el 24 de Julio de 2019, de file:///E:/ /articulos-tesis/GARB6.pdf

Heckman MA, S. K. ((2010)). Energy drinks: An assessment of their market size consumer demographics ingredient profile functionality and regulations in the United States. Comp Rev Food Sci F9:303-307.

Herrera, F. T., Delgado, A. A., Ramírez, C. P., Licea de Anda, E. A., Moreno, C. M., Machuca, Ch. C., (2014). Estudio de la composición proximal de variedades de garbanzo (cicer arietinum 1.) Costa 2004 y blanoro. Ciencia y Tecnología Agropecuaria; Vol. 2. Núm. 2, 915.

Huamani, Q. W. (2015). "Estudio de Compuestos Bioctivos del Aceite Esencial de Muña (Minthostachys. Acobamba - Huancavelica -Perú: Universidad Nacional De Huancavelica.

Huari, G. G. (2014). Efecto Antibacteriano IN VITRO del Aceite Esencial de Minthostachys mollis (MUNAA) EN Streptococcus mutans. lima: Universidad Nacional Mayor de San Marcos.

infoAgro. (06 de agosto de 2002). infoAgro.com. Obtenido de infoAgro.com: http://www.infoagro.com/documentos/el_cultivo_del_garbanzo.asp

Jiménez, C. L. (2017). Escalamiento de la producción de bebidas funcionales a partir de productos vegetales no tradicionales. Bogotá: Universidad Nacional de Colombia Facultad de Ciencias Agrarias.

Lara, Z. S. (2016). Proceso de obtención de Harina de Garbanzo (Cicer arietinum L.) y su utilización en la elaboración de pan de Molde. Quito: Universidad Tecnológica Equinoccial.

Lopez, A. M. (2016). Elaboración de tres embutidos a base de Soja Texturizada y Garbanzo. Tuxtla Gutiérrez, Chiapas: Universidad de Ciencias y Artes de Chiapas. Recuperado el 24 de Julio de 2019, de file:///E:/ /articulos-tesis/TESIS\%20EMBUTIDO\%20GARBANZO.pdf

Martínez, O. Danilo. (2019). Utilización de garbanzo (Cicer arietinum L.) y muña (minthostachys mollys) para la elaboración de una bebida funcional. Universidad Nacional de Chimborazo. 
ISSN: 2600-5859

Quezada, T. K. (2014). "Elaboración de una bebida funcional tipo "Refrescante" A Base de linaza saborizada con piña: Estudio de vida útil y Aporte Nutricional de la formulación". Machala- El Oro- Ecuador: Universidad Técnica De Machala.

Sánchez, S. (2019). El poderoso motivo por el que dejarás de tomar bebidas azucaradas. Obtenido de https://www.alimente.elconfidencial.com/consumo/2019-06-14/motivo-dejar-bebidasazucaradas_2066318/

Seminario, D. (9 de septiembre de 2018). ArtéBlend. Obtenido de ArtéBlend: https://arteblend.com/muna-la-menta-de-los-andes/

Silva, J. I., Cerda, R. B., \& Hernández, M. C. (2013). Uso de Tecnologías Emergentes para la Elaboración de Bebidas Funcionales (aloe vero). Revista Científica de la Universidad Autónoma de Coahuila, $13 . \quad$ Obtenido de http://www.posgradoeinvestigacion.uadec.mx/AQM/No.\%209/3.-\%20bebidas.pdf

USDA. (2010). Antioxidantes and Heatlh. ACES publications.4 pp.

Yapuchura, M. R. (2010). Estudio de los componentes antioxidantes de las hojas de Muña (Minthostachys mollis (Kunth) Griseb.) e Inca Muña (Clinopodium bolivianum (Benth.) Kuntze). Lima: Universidad Nacional Agraria la Molina. 
ISSN: 2600-5859

Vol. 3, N¹.1, p. 427-443, febrero, 2020

\section{PARA CITAR EL ARTÍCULO INDEXADO}

Marcatoma Tixi, J. A., Moposita Vásquez, D. D., Ricaurte Ortiz, P. S., \& Rodas Espinoza, S. L. (2020). Análisis estadístico para la elaboración de una bebida natural no convencional a partir de harina de garbanzo (Cicer Arietinum L.) y hojas de muña (Minthostachys Mollys) . ConcienciaDigital, 3(1.1), 427-443. https://doi.org/10.33262/concienciadigital.v3i1.1.1158

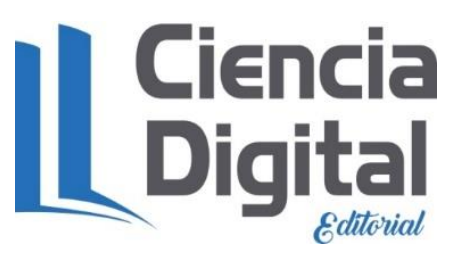

El artículo que se publica es de exclusiva responsabilidad de los autores y no necesariamente reflejan el pensamiento de la Revista Conciencia Digital.

El artículo queda en propiedad de la revista y, por tanto, su publicación parcial y/o total en otro medio tiene que ser autorizado por el director de la Revista Conciencia Digital.
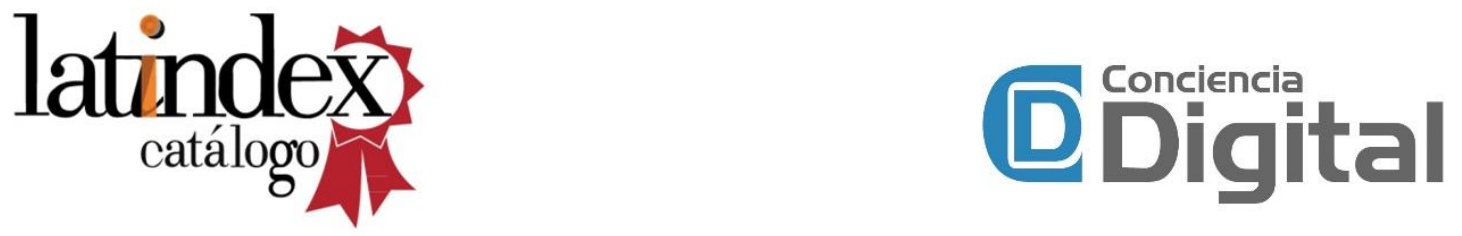